\title{
The maximum luminosity of fast radio bursts
}

\author{
Wenbin $\mathrm{Lu}^{\oplus 1,2 \star}$ and Pawan Kumar ${ }^{2}$ \\ ${ }^{1}$ TAPIR, Walter Burke Institute for Theoretical Physics, Mail Code 350-17, Caltech, Pasadena, CA 91125, USA \\ ${ }^{2}$ Department of Astronomy, University of Texas at Austin, Austin, TX 78712, USA
}

Accepted 2018 October 17. Received 2018 October 16; in original form 2018 July 17

\begin{abstract}
Under the assumption that fast radio bursts (FRBs) are from coherent curvature emission powered by the dissipation of magnetic energy in the magnetosphere of neutron stars, we show that there is a maximum isotropic equivalent luminosity $L_{\max } \sim(2 \times$ $\left.10^{47} \mathrm{erg} \mathrm{s}^{-1}\right) \min \left(\rho_{6}^{2}, B_{16} \rho_{6}^{4 / 3} v_{9}^{-2 / 3}\right)$, where $\rho_{6}=\rho / 10 \mathrm{~km}$ is the curvature radius of the magnetic field lines near the source region, $B_{16}=B / 10^{16} \mathrm{G}$ is the local magnetic field strength, and $v_{9}=v / \mathrm{GHz}$ is the FRB wave frequency. This is because the electric field responsible for accelerating the emitting particles becomes close to the quantum critical strength and is then quickly shielded by Schwinger pairs within a nano-second. Future observations should be able to measure this cut-off luminosity and hence provide a unique probe of the source location and magnetic field strength. We discuss the effects of $L_{\max }$ on the observed flux distributions for repeating bursts from the same object and for the entire FRB population.
\end{abstract}

Key words: stars: neutron-radio continuum: general.

\section{INTRODUCTION}

A major breakthrough in understanding the nature of fast radio bursts (FRBs, Lorimer et al. 2007; Thornton et al. 2013) came when the repeater FRB121102 was precisely localized to be in a dwarf galaxy at redshift $z=0.193$ (Spitler et al. 2014, 2016; Bassa et al. 2017; Chatterjee et al. 2017; Marcote et al. 2017; Tendulkar et al. 2017). Confirmation of the cosmological origin of FRBs means that they are highly energetic events seen in the radio band. The bursts from the repeater show a power-law distribution of isotropic equivalent luminosities $\mathrm{d} N / \mathrm{d} L \propto L^{-\beta}$ in the range from $\sim 10^{40}$ to $\sim 10^{43} \mathrm{erg} \mathrm{s}^{-1}$ and $\beta \sim 1.7$ (Scholz et al. 2016; Hardy et al. 2017; Law et al. 2017; Oostrum et al. 2017). The luminosity distribution of other so-far non-repeating FRBs is less certain due to poor localization and unknown distances. If the Milky Waysubtracted dispersion measures (DMs) are dominated by the intergalactic medium, their isotropic equivalent luminosities range from $\sim 10^{42.5}$ to $\sim 10^{44.5} \mathrm{erg} \mathrm{s}^{-1}$ (see the FRB catalogue by Petroff et al. 2016), with FRB160102 (Bhandari et al. 2018) and FRB170107 (Bannister et al. 2017) being the brightest ones detected so far. We note that these luminosities may not correspond to intrinsic values because (i) the reported peak fluxes in most cases are based on the assumption that the burst occurred at the beam centre, (ii) many FRBs are temporarily broadened due to multipath propagation (Ravi 2019), and (iii) lensing by plasma structures in the host galaxy could further introduce magnification biases (Cordes et al. 2017).

*E-mail: wenbinlu@ caltech.edu
Many models have been proposed to explain FRBs based on considerations of their event rate, duration, and energetics. They generally fall into two categories (see Katz 2016, 2018, for recent reviews of these models): emission from a relativistic outflow which dissipates its energy at large distances from the central compact object (a black hole or neutron star); emission from the magnetospheric plasma of a neutron stars (NS). The high brightness temperatures $T_{\mathrm{b}} \gtrsim 10^{35} \mathrm{~K}$ of FRBs mean that the emission mechanism must be coherent. Lu \& Kumar (2018) showed that models in the first category, i.e. an outflow undergoing internal dissipation or interacting with the surrounding medium, cannot reach typical FRB brightness temperatures before the waves lose energy by induced Compton scattering. On the other hand, if FRBs are produced within the magnetosphere of NSs, the emission process is most likely powered by the dissipation of magnetic energy, instead of rotational energy (Lyutikov 2017; Metzger, Berger \& Margalit 2017).

The energy density of the FRB electromagnetic (EM) waves at radius $r$ from the source (in the limit $r \gg$ source size) is $U_{\mathrm{EM}}=$ $L /\left(4 \pi r^{2} c\right)$, where $L$ is the isotropic equivalent luminosity, and $c$ is the speed of light. The magnetospheric B-field configuration at radius $r \gg R_{*}\left(R_{*} \approx 10 \mathrm{~km}\right.$ being the NS radius) is largely dipolar $B(r) \simeq B_{*}\left(r / R_{*}\right)^{-3}$, where $B_{*}$ is the surface dipolar field. We require the energy density of the B-field $B^{2} / 8 \pi$ to be higher than $U_{\mathrm{EM}}$ and obtain an upper limit for the radius of emission

$r \lesssim\left(6.2 \times 10^{7} \mathrm{~cm}\right) B_{*, 15}^{1 / 2} L_{45}^{-1 / 4}$,

where $B_{*, 15}=B_{*} / 10^{15} \mathrm{G}$ and we use the highest inferred isotropic equivalent luminosity of $L=10^{45} L_{45} \mathrm{erg} \mathrm{s}^{-1}$ as a fiducial value (Bannister et al. 2017; Bhandari et al. 2018). If the EM waves are powered only by particles' kinetic energy, the number density needs 
to be extremely high $n \gtrsim\left(3 \times 10^{25} \mathrm{~cm}^{-3}\right) L_{45} r_{7}^{-2} \gamma^{-1}\left(\mathrm{~m} / \mathrm{m}_{\mathrm{e}}\right)^{-1}$, where $r_{7}=r / 10^{7} \mathrm{~cm}, \gamma$ is the mean Lorentz factor, and $m / m_{\mathrm{e}}$ is the rest mass of the particles divided by electron mass. For any reasonable Lorentz factor, this number density would make the source plasma extremely optically thick due to free-free and/or curvature absorption (Ghisellini \& Locatelli 2018; Kumar, Lu \& Bhattacharya 2017) and radio waves cannot escape. To circumvent this problem, we assume that the FRB waves are emitted by the coherent curvature process when particles are continuously accelerated by a quasi-static E-field parallel to the local magnetospheric B-field, following Kumar et al. (2017).

In this Letter, we show that FRBs should have a maximum luminosity $L_{\text {max }}$ because this parallel E-field must not exceed $\sim 5$ per cent of the quantum critical field $E_{\mathrm{c}}=m_{\mathrm{e}}^{2} c^{3} /(e \hbar) \simeq 4.4 \times 10^{13} \mathrm{esu}$, where $m_{\mathrm{e}}$ and $e$ are the electron mass and charge, and $\hbar$ is the reduced Planck's constant. Since the strength of the parallel E-field depends on the location of the source plasma in the magnetosphere, we can use $L_{\max }$ to constrain the source properties. In Section 2, we derive the upper limit of the parallel E-field and then calculate the maximum luminosity of FRBs. In Section 3, we discuss the effects of the maximum luminosity on the observed flux distributions for repeating bursts from the same object and for the entire population of FRBs. In Section 4, we discuss some caveats of our simplified picture. Our main conclusions are summarized in Section 5. We use CGS units and the convention $Q=10^{n} Q_{n}$. All luminosities are in the isotropic equivalent sense, unless otherwise explicitly stated. We use the Planck best-fitting cosmology (Planck Collaboration 2016).

\section{LUMINOSITY UPPER LIMIT DUE TO SCHWINGER PAIR PRODUCTION}

We consider the situation of a quasi-static and uniform E-field and B-field near the surface of a strongly magnetized NS, with $B \gg E$ and $\boldsymbol{E} \cdot \boldsymbol{B} / B \ll E_{\mathrm{c}}$. It is possible to find an inertial frame where the E-field is parallel to the B-field by applying a non-relativistic Lorentz transform (in the original $\boldsymbol{E} \times \boldsymbol{B}$ direction). In this new frame, the B-field strength is nearly unchanged and the E-field strength is given by $E_{\|} \simeq \boldsymbol{E} \cdot \boldsymbol{B} / B$. It is well known that, when $E_{\|} / E_{\mathrm{c}} \gtrsim 5$ per cent, the E-field will get quickly shielded by copious Schwinger pairs and most of the energy in the E-field gets converted into kinetic/rest-mass energy of pairs (Sauter 1931; Heisenberg \& Euler 1936; Schwinger 1951). For completeness reason, we first rederive the limiting E-field strength (following Stebbins \& Yoo 2015) and then discuss the implications on the maximum FRB luminosity.

The volumetric rate of pair production is given by (e.g. Kim \& Page 2006; Ruffini, Vereshchagin \& Xue 2010)

$\Gamma \simeq \alpha B E_{\|} /(\pi \hbar) \operatorname{coth}\left(\pi B / E_{\|}\right) \exp \left(-\pi E_{\mathrm{c}} / E_{\|}\right)$,

where $\alpha \simeq 1 / 137$ is the fine structure constant and $\operatorname{coth}\left(\pi B / E_{\|}\right) \simeq$ 1 when $E_{\|} \ll \pi B$. Since $\partial^{2} E_{\|} / \partial t^{2}=-4 \pi \partial J / \partial t \simeq-8 \pi e c \Gamma$ (where $J$ is the current density), the time-scale over which the E-field is shielded is given by $\Delta t \simeq(\hbar / \sec \alpha B)^{1 / 2} \exp \left(\pi E_{\mathrm{c}} / 2 E_{\|}\right)$. When $E_{\|}$ $\ll E_{\mathrm{c}}$, this time-scale is an extremely sensitive function of $E_{\text {ll }}$, and the limiting E-field is

$E_{\|, \lim } \simeq \frac{\pi E_{\mathrm{c}}}{\ln \left(8 e c \alpha B \Delta t^{2} / \hbar\right)} \simeq \frac{2.5 \times 10^{12} \mathrm{esu}}{1+0.018 \ln \left(\Delta t_{-9}^{2} B_{15}\right)}$,

where $\Delta t_{-9}=\Delta t / 1 \mathrm{~ns}$ and $B_{15}=B / 10^{15} \mathrm{G}$. We can see that the parallel E-field is quickly shielded on sub-ns time-scale when the parallel E-field exceeds $2.5 \times 10^{12}$ esu.
In the following, we use simple arguments based on energy conservation and source coherence to show that the strength of the parallel E-field is directly related to the FRB luminosity. To generate waves of frequency $v$, the maximum source length in the longitudinal direction is $\sim \bar{\lambda} \equiv c /(2 \pi v)$ in the NS rest-frame. Consider a source of longitudinal size $\lambda$ and transverse size $\ell_{\perp}$, and moving along the local magnetospheric B-field towards the observer at a Lorentz factor $\gamma$ in the NS rest-frame. The local curvature radius of the B-field line is denoted as $\rho$. For a fixed line of sight, the radiation formation length in the NS rest-frame is $\rho / \gamma$, which corresponds to radiation formation time of $\rho /\left(\gamma^{2} c\right)$ in the comoving frame of the source. During this time, the EM fields or the influence by one particle on another travels a distance of $\rho / \gamma^{2}$ in the comoving frame, so the transverse size of the source (which is the same in the comoving frame and NS rest-frame) is limited by

$\ell_{\perp} \lesssim \rho / \gamma^{2}$

The emitting power of the source in the NS rest-frame is a factor of $\sim \gamma^{-4}$ smaller ${ }^{1}$ than the isotropic equivalent luminosity $L$ seen by the observer. This emitting power is supplied by $N \sim n \lambda \ell_{\perp}^{2}$ particles in the coherent volume, where $n$ is the number density of radiating particles in the NS rest-frame. From energy conservation, the emitting power of each particle in the NS rest-frame is given by $E_{\|} e c$. Thus, we obtain

$\gamma^{-4} L \sim n \lambda \ell_{\perp}^{2} E_{\|} e c$, or $L \sim(n e \lambda)\left(\ell_{\perp} \gamma^{2}\right)^{2} E_{\|} c$.

Since all radiating particles in the coherent volume are of the same charge sign (we ignore other background particles that do not contribute to the observed FRB waves), we require that their Coulomb field does not exceed and shield the parallel E-field - the source of energy, i.e.

$n e \lambda \lesssim E_{\|}$

We insert inequalities (4) and (6) into equation (5) and obtain

$L \lesssim E_{\|}^{2} \rho^{2} c$.

Using the upper limit of the parallel E-field $E_{\text {lim }}$, we obtain the maximum isotropic equivalent luminosity of an FRB

$L<L_{\max , 1} \sim\left(2 \times 10^{47} \mathrm{erg} \mathrm{s}^{-1}\right) \rho_{6}^{2}$.

We note that above maximum luminosity has no dependence on the Lorentz factor of the emitting particles. Below, we show that there is another Lorentz-factor-dependent maximum luminosity.

We assume that the emitting particles move close to the speed of light $(\gamma \gg 1)$ along the magnetospheric B-field, and hence there is a current density nec parallel to the B-field. This current induces a transverse magnetic field $B_{\text {ind }} \sim n e \ell_{\perp}$, which must not perturb (or twist) the original B-field by more than a fraction of $\gamma^{-1}$ (the beaming angle):

$n e \ell_{\perp} \lesssim B / \gamma$

We insert the above inequality into equation (5) and obtain

$L \lesssim B E_{\|} \gamma \lambda\left(\ell_{\perp} \gamma^{2}\right) c \lesssim B E_{\|} \gamma \lambda \rho c$

where equation (4) has been used in the second step. In the coherent curvature emission model, the radiation formation length is $\rho / \gamma \simeq$ $\gamma^{2} \lambda$ in the NS rest-frame, so we obtain the typical Lorentz factor of

${ }^{1} \mathrm{~A}$ factor of $\gamma^{-2}$ comes from relativistic beaming, and another factor of $\gamma^{-2}$ is because the difference between the speeds of photons and emitting particles is $\sim c / \gamma^{2}$ in the limit $\gamma \gg 1$. 
emitting particles to be $\gamma \simeq(\rho / \hbar)^{1 / 3}$. We plug this Lorentz factor into equation (10) and make use of $E_{\|}<E_{\|, \text {lim }}=2.5 \times 10^{12} \mathrm{esu}$, and then obtain

$L \lesssim L_{\max , 2} \sim\left(2 \times 10^{46} \mathrm{erg} \mathrm{s}^{-1}\right) B_{15} \rho_{6}^{4 / 3} v_{9}^{-2 / 3}$,

where $B_{15}=B / 10^{15} \mathrm{G}$ is the B-field strength in the source region and $v_{9}=v / \mathrm{GHz}$. The strongest B-fields of NSs are believed to be produced due to amplification by the $\alpha-\Omega$ dynamo and may reach a few $\times 10^{17} \mathrm{G}$, limited by the energy budget of the differential rotation (Thompson \& Duncan 1993). Thus, the inequality (11) may be weaker than (8). Nevertheless, we combine these two conditions and obtain

$$
L \lesssim L_{\max } \sim\left(2 \times 10^{47} \mathrm{erg} \mathrm{s}^{-1}\right) \min \left(\rho_{6}^{2}, B_{16} \rho_{6}^{4 / 3} v_{9}^{-2 / 3}\right)
$$

\section{OBSERVATIONS}

In this section, we discuss the effects of $L_{\max }$ on the observed flux distributions for repeating bursts from the same object and for the entire population of FRBs.

\subsection{Repeating bursts from the same object}

The luminosity function for the repeater FRB121102 is a power law with $\beta=1.7_{-0.5}^{+0.3}$ (Law et al. 2017) extending from $\sim 10^{40}$ to $\sim 10^{43} \mathrm{erg} \mathrm{s}^{-1}$, with (so-far) the brightest one having peak flux $S=$ $24 \pm 7$ Jy (Oostrum et al. 2017). One possible scenario is that the bursts are produced near the surface of a NS where $\rho \sim 10 \mathrm{~km}$ and that the B-field strength near the source is $\sim 10^{15} \mathrm{G}$ (typical dipole surface field strength inferred from Galactic magnetars). In this case, we have $L_{\max } \sim 10^{46} \mathrm{erg} \mathrm{s}^{-1}$ and the observed flux distribution should have a cut-off at $\sim 10^{4} \mathrm{Jy}$. Note that, if the bursts are produced far-away from the NS surface (but within the light cylinder), then the B-field strength in the source region is much weaker than that near the surface, and hence the cut-off should show up at a lower flux level $\ll 10^{4} \mathrm{Jy}$. In the future, we may detect more repeaters, and then an interesting possibility is that each repeating source may have different B-field strength and curvature radius and hence different $L_{\max }$. We can see that the cut-off luminosity $L_{\max }$ provides a powerful probe of the emission location and the B-field strength near the source.

\subsection{The entire FRB population}

To show the observational effects of $L_{\max }$ for the entire FRB population, we assume a global power-law luminosity function in the form

$\frac{\mathrm{d} \dot{N}}{\mathrm{~d} L}=\Phi(z)(\beta-1) L_{0}^{\beta-1} L^{-\beta}$,

where $L_{0}$ is a (fixed) reference luminosity, $\beta$ is the power-law index, and $\Phi(z)$ is the normalization including the cosmological evolution. We do not assume that all FRBs repeat the same way as FRB121102, so the global power-law index may not be the same as the repeater. Effectively, we treat each repetition as a separate FRB originated from the same redshift. We leave $\beta$ as a free parameter between 1 and 2.5. The lower and upper limits are motivated by the observations that brighter bursts are rarer than dimmer ones and that the DM distribution of known FRBs is not concentrated near the lowest end. For simplicity, we also assume that FRBs (on average) have a flat spectrum near $\sim \mathrm{GHz}$ frequencies (Gajjar et al. 2018), otherwise a receiver operating at a certain frequency band will observe different parts of the intrinsic spectrum for sources at different redshifts. This complication can be effectively included in the $\Phi(z)$ factor and does not significantly affect our calculations below.

In the ideal case of no propagation effects such as scattering broadening, plasma lensing, absorption, and gravitational lensing (these complications will be discussed in Section 4), the flux distribution of the observed bursts is

$$
\begin{aligned}
\dot{N}_{\text {det }}(>S)= & \int_{0}^{z_{\max }} \frac{\mathrm{d} z}{1+z} \frac{\mathrm{d} V}{\mathrm{~d} z} \int_{4 \pi D_{\mathrm{L}}^{2} S}^{L_{\max }} \frac{\mathrm{d} \dot{N}}{\mathrm{~d} L} \mathrm{~d} L \\
= & \int_{0}^{z_{\max }} \frac{\mathrm{d} z}{1+z} \frac{\mathrm{d} V}{\mathrm{~d} z} \Phi(z) \max [0, \\
& \left.\left(\frac{4 \pi D_{\mathrm{L}}^{2} S}{L_{0}}\right)^{1-\beta}-\left(\frac{L_{\max }}{L_{0}}\right)^{1-\beta}\right],
\end{aligned}
$$

where $z_{\max }$ is the maximum redshift at which FRBs can be produced, $\mathrm{d} V / \mathrm{d} z$ is the differential comoving volume within the field of view for a certain telescope, $D_{\mathrm{L}}(z)$ is the luminosity distance for redshift $z$, and $L_{\max }$ is the maximum isotropic equivalent luminosity of FRBs as given by equation (12). In the limit $L_{\max } \rightarrow \infty$, the $\left(L_{\max } / L_{0}\right)^{1-\beta}$ term in equation (14) vanishes, so we obtain a power-law flux distribution $\dot{N}_{\text {det }}(>S) \propto S^{1-\beta}$, independent of the cosmological evolution of FRB rate $\Phi(z)$. This is because the redshift distribution of bursts in each flux bin $[S, S+\mathrm{d} S]$ is independent of $S$. The flux distribution of the observed FRBs from Parkes telescope is consistent with a single power law but the power-law index is not well constrained (see the discussions by Vedantham et al. 2016; Bhandari et al. 2018; Macquart \& Ekers 2018), due to the lack of a homogeneous sample with sufficient number of bursts. For the luminosity function in equation (13), since $\beta>1$, bursts near the cut-off luminosity are very rare and the only way to increase their detection rate is to use telescopes with larger field of views.

The critical flux at which the two terms on the RHS of equation (14) equal is given by

$S_{\mathrm{c}}=\left[\frac{\int_{0}^{z_{\max }} \frac{\mathrm{d} z}{1+z} \frac{\mathrm{d} V}{\mathrm{~d} z} \Phi(z)\left(4 \pi D_{\mathrm{L}}^{2}\right)^{1-\beta}}{\int_{0}^{z_{\max }} \frac{\mathrm{d} z}{1+z} \frac{\mathrm{d} V}{\mathrm{~d} z} \Phi(z)}\right]^{\frac{1}{\beta-1}} L_{\max }$,

which is linearly proportional to $L_{\max }$ and depends on the power-law index $\beta$, the cosmological rate evolution $\Phi(z)$, and the maximum redshift $z_{\max }$. At flux levels much below $S_{\mathrm{c}}$, the flux source count is a power-law $\dot{N}_{\text {det }}(>S) \propto S^{1-\beta}$, but above this flux level, the deficit of FRBs with $L \gtrsim L_{\max }$ will be seen as a break in the observed flux distribution. From equation (14), one can show that the distribution at $S \gg S_{\text {c }}$ approaches $N(>S) \propto S^{-1.5}$ (Euclidean), since bursts with $L \sim L_{\max }$ from the nearby Universe will dominate. In Fig. 1, we show the critical flux level $S_{\mathrm{c}}$ as a function of the power-law index for four different cases: (i) FRB rate $\Phi(z)$ either tracks the cosmic star-formation history or is non-evolving throughout the history; (ii) the maximum redshift $z_{\max }$ is either 2 or 6 . The choices of $z_{\max }$ is motivated by the highest redshift of $z \sim 2$ inferred from the DM of FRB 160102 (Bhandari et al. 2018). We find that the value of $S_{\mathrm{c}}$ has a weak dependence on the cosmic evolution of FRB rate and that the dependence on the power-law index $\beta$ is also fairly mild (varying by about one order of magnitude).

Therefore, for a power-law global luminosity function, we predict the cumulative flux distribution to be $\dot{N}_{\text {det }}(>S) \propto S^{1-\beta}$ below the flux level $S_{\mathrm{c}} \sim\left(10^{3}-10^{4}\right) L_{\max , 47}$ Jy and $\dot{N}_{\text {det }}(>S) \propto S^{-1.5}$ at $S \gg S_{\mathrm{c}}$. The deficit of high flux FRBs should be noticeable with sufficiently large number of detections near and above $S \sim S_{\mathrm{c}}$. The cut-off luminosity $L_{\max }$ can be inferred from the critical flux $S_{\mathrm{c}}$ via equation (15). 


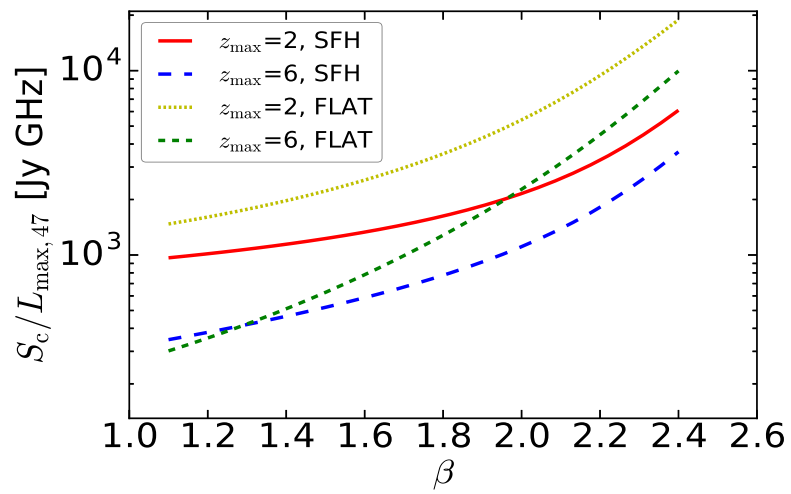

Figure 1. The critical flux $S_{\mathrm{c}}$ (equation 15) as a function of the power-law index of the global luminosity function, for four different cases. For the red (solid) and blue (long-dashed) curves, we assume that the FRB rate tracks the cosmic star-formation history (SFH) given by Madau \& Dickinson (2014). For the yellow (dotted) and green (short-dashed) curves, we assume a nonevolving (FLAT) FRB rate history. Two choices of maximum redshifts are shown $z_{\max }=2$ and 6 .

Unfortunately, the expected all-sky rate of FRBs near $S_{\text {c }}$ is highly uncertain, mainly because the power-law index $\beta$ is only weakly constrained by current data. From the Parkes FRB sample, Bhandari et al. (2018) inferred a $\operatorname{rate}^{2}$ of $\dot{N}_{\text {det }}\left(\gtrsim S_{\text {th }}\right) \sim$ $2 \times 10^{3} \mathrm{sky}^{-1} \mathrm{~d}^{-1}$ above the completeness threshold flux $S_{\mathrm{th}} \sim$ $3 \mathrm{Jy} \mathrm{GHz}$. Taking their rate at face value, we expect the all-sky rate near the flux level $S_{\mathrm{c}} \sim 3 \times 10^{3} L_{\max , 47} \mathrm{Jy} \mathrm{GHz}$ to be $\dot{N}_{\exp }\left(\gtrsim S_{\mathrm{c}}\right) \sim$ $2 \times 10^{3(2-\beta)} L_{\max , 47}^{1-\beta} \mathrm{sky}^{-1} \mathrm{~d}^{-1}$. For $L_{\max }=10^{47} \mathrm{erg} \mathrm{s}^{-1}$ and $\beta=1.7$ (or 2.3), the product of solid angle and observing time per FRB detection with $S \sim S_{\mathrm{c}}$ is $\sim 20 \mathrm{sr} \cdot \mathrm{h}$ (or $1.2 \times 10^{3} \mathrm{sr} \cdot \mathrm{h}$ ).

\section{DISCUSSION}

In this section, we discuss some caveats of our simplified picture. As more data accumulates, they may become important issues to look at in detail in future works.

(1) The signal-to-noise ratio of an FRB is determined by a combination of flux $S$ and duration $\tau$ as SNR $\propto S \sqrt{\tau}$ (Keane \& Petroff 2015). In equation (14), the luminosity at the detection threshold $4 \pi D_{\mathrm{L}}^{2} S$ (for a given redshift and flux) should include an additional factor $\propto \tau^{-1 / 2}$ and then we integrate over the intrinsic distribution of burst durations. We can see that the shape of the flux distribution function $\mathrm{d} \dot{N}_{\text {det }} / \mathrm{d} S$ stays the same, as long as the intrinsic distribution of burst durations is not correlated with their luminosities (such a correlation has not been found in the literature).

(2) The observed flux $S_{\text {obs }}$ may be different from the intrinsic/unattenuated flux $S=L / 4 \pi D_{\mathrm{L}}^{2}$ for a given redshift and luminosity. When there is significant scattering broadening, intrachannel dispersion smearing, absorption, insufficient time resolution, or when the location of the burst is far away from the centre of

\footnotetext{
${ }^{2}$ We note that the reported fluxes in their sample are based on the assumption that the bursts occurred within the half-power width of the discovery beam. It was later realized that, at discovery, FRB121102 (the repeater) was in a side lobe where the sensitivity is $\sim 10$ percent of that at the beam centre (Spitler et al. 2014; Chatterjee et al. 2017). Thus, the locations of some Parkes bursts may also be in the side lobes and hence their true fluxes are higher than those reported. Since the effective field of view (including side lobes) is larger, this will give a lower all-sky rate $\dot{N}_{\text {det }}\left(\gtrsim S_{\text {th,eff }}\right)$ above a higher effective completeness threshold flux $S_{\text {th, eff. }}$
}

the discovery beam, we have $S_{\text {obs }}<S$. On the other hand, magnification bias due to lensing of FRBs by plasma structures in the host galaxies (Cordes et al. 2017) may lead to $S_{\mathrm{obs}}>S$ for a fraction of the observed bursts. Thus, the critical flux above which the luminosity function cut-off is noticeable in the source count may be different than the unattenuated flux $S_{\mathrm{c}}$ in equation (15). These effects make it harder to infer the maximum luminosity $L_{\max }$ from observations, but the existence of a cut-off in the luminosity function can still be tested.

(3) We have assumed the luminosity function to be a single power law with a cut-off at $L_{\max }$ and the power-law index to be in the range $(1,2.5)$. For other luminosity function models, equations (14) and (15) are generally valid. For instance, an alternative luminosity function is a broken power law and in this case $\beta \geq 2.5$ is allowed on the high-luminosity end (as is the case of long gamma-ray bursts). Another possibility is that there is another cut-off at the low luminosity end. In these cases, it is straightforward to solve equation (15) for the critical flux $S_{\mathrm{c}}$ (which may be significantly different from that shown in Fig. 1) and determine where the deficit of high-luminosity FRBs above $L_{\max }$ will show up in the observed flux distribution $\mathrm{d} \dot{N}_{\text {det }} / \mathrm{d} S$.

(4) The observed flux distribution suffers from magnification bias due to strong gravitational lensing by intervening galaxies. For FRBs at $z \sim 2$ (near the peak of the cosmic star-formation history), the optical depth for large magnification $\mu \gg 1$ is roughly $P(>\mu) \sim 10^{-3} \mu^{-2}$ (e.g. Takahashi et al. 2011), which should be multiplied by the luminosity function $\mathrm{d} \dot{N} / \mathrm{d} \ln L \propto L^{1-\beta}$ to calculate the contribution to the source count at a given flux. If $\beta<3$, then the majority of the lensed sources with apparent luminosity $\gg L_{\max }$ come from those sources with intrinsic luminosity $L \sim L_{\max }$ (Blandford \& Narayan 1992). Thus, the observed flux distribution of lensed (L) FRBs should be $\dot{N}_{\text {det, }}(>S) \propto S^{-2}$ above the critical flux $S_{\text {c }}$, which is steeper than $\dot{N}_{\text {det, NL }}(>S) \propto S^{-1.5}$ for unlensed (NL) FRBs at $S \gg S_{\text {c }}$. Therefore, the unlensed population always dominates at all flux levels and magnification bias should not a serious problem for constraining the cut-off luminosity $L_{\max }$.

\section{SUMMARY}

In this Letter, we provide a novel way to test the model that FRBs are from coherent curvature emission powered by the dissipation of magnetic energy in the magnetosphere of NSs. In this model, the emitting particles are continuously accelerated by a quasi-static E-field parallel to the local B-field. We use simple arguments based on energy conservation and source coherence to show that the isotropic equivalent luminosity of an FRB is directly related to the parallel E-field strength. When this parallel E-field exceeds about 5 per cent of the quantum critical field strength, it is quickly shielded by Schwinger pairs on sub-ns time-scales (and hence the FRB emission cannot be sustained). Based on this limiting E-field, we show that there is a maximum isotropic equivalent luminosity of $L_{\max } \sim\left(2 \times 10^{47} \mathrm{erg} \mathrm{s}^{-1}\right) \min \left(\rho_{6}^{2}, B_{16} \rho_{6}^{4 / 3} v_{9}^{-2 / 3}\right)$, where $\rho$ is the curvature radius of the magnetic field lines near the source region. Future observations can measure $L_{\max }$ and hence probe the source location and B-field strength.

For the repeater FRB121102, this cut-off luminosity corresponds to a maximum flux of $S_{\max }=L_{\max } / 4 \pi D_{\mathrm{L}}^{2} \sim 10^{5} L_{\max , 47} \mathrm{Jy}$. Each repeating source may have a different $L_{\max }$ from the others, depending on the source location and B-field strength. We encourage monitoring the repeater for an extended amount of time with a low-sensitivity telescope. 
If the entire population of FRBs has a global luminosity function, then the cut-off luminosity $L_{\max }$ should be observable as a deficit of high-flux FRBs in the observed flux distribution. Taking the simplest case of a power-law luminosity function $\mathrm{d} N / \mathrm{d} L \propto L^{-\beta}$ as an example, we show that there is a critical flux $S_{\mathrm{c}} \sim\left(10^{3}-10^{4}\right) L_{\text {max }, 47} \mathrm{Jy}$, below and above which the cumulative flux distribution will be $\dot{N}_{\text {det }}(>S) \propto S^{1-\beta}$ (for $S \ll S_{\mathrm{c}}$ ) and $\dot{N}_{\text {det }}(>S) \propto S^{-1.5}$ (for $S \gg S_{\mathrm{c}}$ ). Bright FRBs near or above the critical flux $S_{\mathrm{c}}$ have a much lower all-sky rate than those currently detected. Extrapolating the rate of Jy-level FRBs to higher fluxes and assuming $L_{\max }=10^{47} \mathrm{erg} \mathrm{s}^{-1}$, we estimate the detection rate of bright FRBs near $S_{\mathrm{c}}$ by ASKAP (sky coverage $\Omega / 4 \pi \sim 4 \times 10^{-3}$ at $0.7-1.8 \mathrm{GHz}$, Bannister et al. 2017) to be $0.06 \mathrm{~d}^{-1}$ for $\beta=1.7$ and $0.001 \mathrm{~d}^{-1}$ for $\beta=2.3$. The rate for CHIME (sky coverage $\Omega / 4 \pi \sim 7 \times 10^{-3}$ at $400-800 \mathrm{MHz}$, The CHIME/FRB Collaboration 2018) may be slightly higher. We encourage searching for ultra-bright FRBs by low-sensitivity telescopes with large field of views.

\section{ACKNOWLEDGEMENTS}

We thank Vikram Ravi for useful discussions. We also thank the referee for comments which improved the clarity of the presentation. This research benefited from interactions at the ZTF Theory Network Meeting, funded by the Gordon and Betty Moore Foundation through Grant GBMF5076. WL was supported by the David and Ellen Lee Fellowship at Caltech.

\section{REFERENCES}

Bannister K. W. et al., 2017, ApJ, 841, L12

Bassa C. G. et al., 2017, ApJ, 843, L8

Bhandari S. et al., 2018, MNRAS, 475, 1427

Blandford R. D., Narayan R., 1992, ARA\&A, 30, 311

Chatterjee S. et al., 2017, Nature, 541, 58

Cordes J. M., Wasserman I., Hessels J. W. T., Lazio T. J. W., Chatterjee S., Wharton R. S., 2017, ApJ, 842, 35
Gajjar V. et al., 2018, ApJ, 863, 2

Ghisellini G., Locatelli N., 2018, A\&A, 613, A61

Hardy L. K. et al., 2017, MNRAS, 472, 2800

Heisenberg W., Euler H., 1936, Zeitschr. Phys., 98, 714

Katz J. I., 2016, Mod. Phys. Lett. A, 31, 1630013

Katz J. I., 2018, PrPNP, 103, 1

Keane E. F., Petroff E., 2015, MNRAS, 447, 2852

Kim S. P., Page D. N., 2006, Phys. Rev. D, 73, 065020

Kumar P., Lu W., Bhattacharya M., 2017, MNRAS, 468, 2726

Law C. J. et al., 2017, ApJ, 850, 76

Lorimer D. R., Bailes M., McLaughlin M. A., Narkevic D. J., Crawford F., 2007, Science, 318, 777

Lu W., Kumar P., 2018, MNRAS, 477, 2470

Lyutikov M., 2017, ApJ, 838, L13

Macquart J.-P., Ekers R. D., 2018, MNRAS, 474, 1900

Madau P., Dickinson M., 2014, ARA\&A, 52, 415

Marcote B. et al., 2017, ApJ, 834, L8

Metzger B. D., Berger E., Margalit B., 2017, ApJ, 841, 14

Oostrum L. C. et al., 2017, Astron. Telegram, 10693

Petroff E. et al., 2016, PASA, 33, e045

Planck Collaboration, 2016, A\&A, 594, A13

Ravi V., 2019, MNRAS, 482, 1966

Ruffini R., Vereshchagin G., Xue S.-S., 2010, Phys. Rep., 487, 1

Sauter F., 1931, Zeitschr. Phys., 69, 742

Scholz P. et al., 2016, ApJ, 833, 177

Schwinger J., 1951, Phys. Rev., 82, 664

Spitler L. G. et al., 2014, ApJ, 790, 101

Spitler L. G. et al., 2016, Nature, 531, 202

Stebbins A., Yoo H., 2015, preprint (arXiv:1505.06400)

Takahashi R., Oguri M., Sato M., Hamana T., 2011, ApJ, 742, 15

Tendulkar S. P. et al., 2017, ApJ, 834, L7

The CHIME/FRB Collaboration, 2018, ApJ, 863, 43

Thompson C., Duncan R. C., 1993, ApJ, 408, 194

Thornton D. et al., 2013, Science, 341, 53

Vedantham H. K., Ravi V., Hallinan G., Shannon R. M., 2016, ApJ, 830, 75

This paper has been typeset from a $\mathrm{T}_{\mathrm{E}} \mathrm{X} / \mathrm{L} \mathrm{T} \mathrm{E} \mathrm{X}$ file prepared by the author. 